\title{
"Minimally invasive" lumbar spine surgery: a critical review
}

\author{
Michael Payer
}

Received: 4 April 2011 /Accepted: 5 April 2011 /Published online: 1 May 2011

(C) Springer-Verlag 2011

\begin{abstract}
Background Minimal-access technology has evolved rapidly with "tubular" or "percutaneous" approaches for decompression and stabilization in the lumbar spine. Potential benefits (smaller scars, diminished local pain, reduced blood loss, reduced postoperative wound pain, shorter hospital stays) have to be weighed against possible drawbacks (reduced orientation, steep learning curve, increased radiation exposure, dependency on technology, cost). While non-comparative case series are often rather enthusiastic, comparative studies and particularly RCTs are scarce and might convey a more realistic appreciation.

Methods A MEDLINE search via PubMed was performed to find all English-language studies comparing "open" or "traditional" or "conventional" with "minimally invasive" or "percutaneous" or "tubular" approaches in degenerative lumbar spine surgery.

Results Only nine comparative studies could be retrieved altogether. No clear benefit could be found for minimally invasive procedures in lumbar disc herniation, TLIF, or PLIF. There seems to be a slight advantage in terms of hardware safety in open procedures.

Conclusions This review, based solely on the very limited number of available comparative studies, shows no relevant benefit from minimally invasive techniques, and a tendency
\end{abstract}

\footnotetext{
M. Payer $(\square)$

Department of Neurosurgery, Klinik Hirslanden,

Witellikerstrasse 40,

8032 Zurich, Switzerland

e-mail: mpayer@hotmail.com

M. Payer

Department of Neurosurgery, University Hospital of Geneva, Geneva, Switzerland
}

for more safety in open procedures in lumbar disc herniation, TLIF and PLIF.

Keywords Spine surgery $\cdot$ Lumbar spine $\cdot$ Minimally invasive $\cdot$ Percutaneous $\cdot$ Tubular $\cdot$ Review
Abbreviations
VAS Visual analogue scale
EBL Estimated blood loss
TLIF Transforaminal lumbar interbody fusion
PLIF Posterior lumbar interbody fusion
ODI Oswestry disability index
LOS Length of stay
RCT Randomized controlled trial

\section{Introduction}

As for many other surgical subspecialties, minimal-access technology has evolved remarkably over the past two decades for spine surgery. Numerous endoscopic, tubular, and percutaneous approaches have been developed for traditional decompression and stabilization or placement of newly developed implants such as spacers and artificial discs. The trend for these less-invasive techniques is understandable, as smaller access should result in smaller scars, diminished local pain, reduced blood loss, reduced postoperative wound pain, and therefore shorter hospital stays with the potential to carry out certain procedures on an outpatient basis [10]. However, minimally invasive procedures have several challenges [13]:

- Three-dimensional anatomical exposure and therefore orientation is considerably reduced; manipulating instruments through small access channels is particularly 
demanding; and complication management may be very difficult and even necessitate conversion to open surgery

- A strong dependency on technical equipment (access instruments, fluoroscopy, navigation) may make minimally invasive procedures more expensive and subject to technical failures

- Placing spinal implants through tubes or "percutaneously" commonly requires high doses of radiation exposure for both the surgeon (team) and the patient

- Technical dependency coupled with increased manual challenges accounts for a very steep (i.e., long and again expensive and often complication-ridden) learning curve.

All of this leads to the fundamental question: Is there a true benefit of so-called "minimally invasive" spinal surgery or is it just a new trend with increased risks?

There are numerous enthusiastic non-comparative case series on minimally invasive spinal surgery, but caution is necessary: in the attempt to celebrate technical advances, both the involved spinal surgeons and their corresponding industry partners may be biased in analyzing "their" new surgical methods.

This critical review is undertaken in an attempt to identify potential advantages or disadvantages of minimally invasive techniques in the most commonly practiced procedures for lumbar degeneration, based exclusively on comparative studies.

\section{Materials and methods}

A MEDLINE search via PubMed was performed to find all English-language studies comparing "open" or "traditional" or "conventional" with "minimally invasive" or "percutaneous" or "tubular" approaches in degenerative lumbar spine surgery. Title-screening with these search terms included all language publications with the date of last search on July 31, 2010. Endoscopic procedures were not included in this review. If the title did not clearly rule out a positive search result, the abstract was screened, and if the abstract did not clearly rule out a positive search result, the article was read through. The identified articles were grouped together for the most common (and thus lumbar) spinal procedures: lumbar disc herniation (LDH), posterior lumbar interbody fusion (PLIF), and transforaminal lumbar interbody fusion (TLIF).

\section{Results}

Only nine comparative studies could be retrieved: two about lumbar disc herniation $[1,6]$, four about TLIF $[3,12$,
14, 16] and three about PLIF $[5,9,11]$. No comparative article could be retrieved about the most common lumbar spine procedure of all, pure lumbar decompression for stenosis.

Lumbar disc herniation

One retrospective and one prospective comparative study were found.

Harrington in his retrospective study compared 35 open with 31 minimally invasive cases (expanding retractor and microscope): While surgical duration, blood loss, complications, and outcome were similar in both groups, pain medication requirements (average dose of hydrocodone $13.4 \mathrm{mg}$ for the minimally invasive group and $20.9 \mathrm{mg}$ for the open group) and hospitalization duration (discharge on the day of surgery in $45 \%$ in the minimally invasive group vs. $6 \%$ in the open group) were less in the minimally invasive group [6].

Arts in his randomized controlled trial found that conventional microdiscectomy with muscle retractor and microscope or loupe magnification in 159 cases resulted in generally equal outcome with slightly better pain results than microscopic disc herniation removal through a tube in 166 cases. The surgeons were familiar with both techniques and concluded that "patients who underwent tubular diskectomy fared worse with regard to leg and back pain and fewer patients reported complete recovery at 1 year". At the final 1-year follow-up, the mean Roland-Morris Disability Questionnaire score was in favor of the "conventional" group (3.4 vs. 4.7 in the "tubular" group), as was improvement on the visual analogue scale for leg and back pain and self-reported recovery $(79 \%$ "good" in the conventional vs. $69 \%$ in the tubular group) [1].

\section{TLIF}

Four retrospective studies and no prospective study were retrieved.

Villavicencio et al., in their retrospective study, compared 63 open with 76 matching minimally invasive cases with a mean follow-up of 37.5 months and found that patients in the open TLIF group had greater VAS improvement and overall satisfaction than the minimally invasive group; mean EBL was less in the minimally invasive group but also low in the open group $(367 \mathrm{cc})$ and hospital stay 1 day shorter in the minimally invasive group. The total rate of neurological deficit was $10.5 \%$ in the minimally invasive TLIF group compared to $1.6 \%$ in the open group. They concluded that "on the basis of the results of this study, it is safe to say that minimally invasive TLIF technique is not superior compared to the open approach. The potential benefits of less blood loss and a faster 
recuperation appear to be offset by a higher rate of neurological complications" [16].

Peng et al. found a similar long-term clinical outcome and fusion rates at 2 years when retrospectively comparing 29 minimally invasive with 29 open TLIF cases; postoperative pain medication was only slightly higher in the open group, mean EBL $150 \mathrm{ml}$ in the minimally invasive and $681 \mathrm{ml}$ in the open group, and surgery time was longer in the minimally invasive group (216 vs. $170 \mathrm{~min}$ ) [12].

Schizas and colleagues document a shorter hospital stay in their initial experience with 18 cases of minimally invasive compared to 18 open TLIFs; they concluded that "no difference was observed in postoperative pain, initial analgesia consumption, VAS or ODI between the groups. Three pseudarthroses were observed in the minimally invasive TLIF group although this was not statistically significant. A steeper learning effect was observed for the minimally invasive TLIF group" [14].

Dhall and coworkers compared 21 cases of mini-open TLIF with average follow-up of 24 months with 21 cases of open TLIF with mean follow-up of 34 months. While EBL (194 cc vs. $505 \mathrm{cc}$ ) and length of stay ( 3 days vs. 5.5 days) were reduced in the mini-open group, a higher rate of hardware-associated complications was found in the miniopen TLIF (one misplaced screw, one cage migration, one pseudarthrosis) compared to the open technique (one misplaced screw) [3].

\section{PLIF}

Two retrospective studies and one prospective cohort study were retrieved.

Ntoukas retrospectively compared 20 minimally invasive with 20 open PLIFs and found "less blood loss, less postoperative pain, quicker recovery and shorter duration of hospitalization. However, in the long run, one year after surgery, both groups showed no significant difference with regards to clinical and radiographic outcome", and "...on despite these benefits, the minimally invasive group also experienced a longer surgical and radiation time as compared to the 'open' group" [9].

Gepstein retrospectively compared 30 cases of minimally invasive PLIF with average follow-up of 29 months with earlier 30 cases of open PLIF for post-discectomy pain. Mean hospitalization was 2.75 days and blood loss negligible in the minimally invasive group, 5.5 days and $750 \mathrm{cc}$ in the open group. Clinical and radiological results were comparable at the mean follow-up of 29 months in the minimally invasive group and 37 months in the open group [5].

Park prospectively documented 32 minimally invasive and 29 open PLIF cases, whereby minimally invasive procedures were not covered by the country's insurance and thus were chosen only by financially "healthy" patients.
The authors found no significant difference between the two groups in clinical and radiographic results with 1-year minimum follow-up. The minimally invasive group had less EBL of $433 \mathrm{cc}$ on average compared to $738 \mathrm{cc}$ in the open group and a shorter hospital stay of 5.3 days compared to 10.8 days in the open group. Whether the financial background played a role in leaving the hospital earlier to return to professional activity earlier must remain open. The minimally invasive group needed longer surgical time with $192 \mathrm{~min}$ vs. $149 \mathrm{~min}$ in the open group and showed two cases of technical complications in the form of misplaced pedicle screw and cage migration [11].

\section{Discussion}

"Minimally invasive" spinal procedures have been (and in many spinal centers still are) in the focus of both the medical technology industry and the involved developing surgeons. Some undebatable advantages may exist in creating reduced access morbidity, but making a compromise on exposure and depending on "heavy" technological equipment may come at a high price in terms of patient safety and finance.

In this review, "traditional" and "minimally invasive" approaches in the most common posterior lumbar procedures were analyzed from available comparative articles. In lumbar disc herniation, no relevant benefit was found in minimally invasive procedures $[1,6]$. Though LOS and perioperative pain medication were reduced in the minimally invasive group in one study, such a retrospective evaluation can be biased as incentives for discharge might have been different for the groups; furthermore, perioperative pain medication differed only so little that there are no relevant consequences in daily practice (average dose of hydrocodone $13.4 \mathrm{mg}$ for the minimally invasive group and $20.9 \mathrm{mg}$ for the open group) [6]. In the single more meaningful prospective study a slight superiority in clinical 1-year outcome could be shown for the "open" procedure [1].

No convincing benefit was found for minimally invasive TLIF compared to open TLIF [3, 12, 14, 16]. The report of higher rates of neurological complications [16], a "steep learning curve" [14], and higher rate of hardware-associated complications [3] for the minimally invasive techniques convey little enthusiasm. The only potential advantage in the minimally invasive TLIF was a reduced EBL in one series [12], which in a retrospective series has limited meaning as more attention on hemostasis is likely to be spent on the "new" minimally invasive technique.

For PLIF, slightly reduced initial postoperative LOS or EBL could be reported for minimally invasive techniques, whereas long-term outcome was not different between open and minimally invasive procedures $[5,9,11]$, However, 
decreased EBL and shorter LOS in the "new minimally invasive" technique again is a weak argument, as the new technique has been compared retrospectively with a historical "open" control group [5]. In the only prospective study, misplaced pedicle screws and cage migration, though rare, were found only in the minimally invasive group [11].

Although this review, based solely on comparative studies, is far from giving answers as to which approach is best for which spinal procedure, it clearly shows not only no relevant benefit from minimally invasive procedures, but even a tendency for safer open surgery.

The current literature review finding is in accordance with Epstein's remarkable comment on minimally invasive cervical laminoforaminotomy: Many neurosurgical operations are already very difficult even with maximal exposure; optimal exposure facilitates maneuvering the microscope and surgical instruments, avoids the movement restrictions of minimally invasive approaches, and helps to limit complications by adequate exposure of neural and vascular structures. He further argues that the true complication rate, on or off the learning curve, might not be available, and that informal colleague discussions reveal disillusion and reverting back to open procedures; the overall aim of spinal surgery is to perform safe and cost-effective procedures [4].

Compared to transthoracic, transabdominal, or arthroscopic procedures, where increased use of minimally invasive surgery is facilitated by anatomically pre-existing cavities, minimally invasive spinal procedures from a posterior approach lack such "key-hole" anatomy and can only be performed by either making compromise on exposure or increasing the use of technological help such as navigation and computer assistance in hardware placement. However, there are several practical concerns with both of these "compensatory" strategies:

- Learning curves are a particular challenge in all surgical specialties, as optimal surgical management requires many years of experience. Investing more time in minimally invasive procedures either prolongs the entire surgical training duration or requires cutting back on traditional techniques, which in turn hurts intraoperative crisis management and decision-making [2]. These deficiencies deserve educational effort at a higher priority than accorded so far.

- Radiation exposure during minimally invasive procedures has not been clearly evaluated so far and may be a concern; though systematic comparative studies are not available, one prospective controlled trial found that minimally invasive lumbar microdiscectomy exposed the surgeon to significantly more radiation than open microdiscectomy [8]. On the other hand, navigationassisted fluoroscopy has also been shown to decrease radiation exposure during minimally invasive spine surgery in the case of TLIF; however, as this was a cadaver study, interpretation is limited [7].

- The recent explosion of innovative technology in minimally invasive spine surgery, coupled with navigation, creates new cost. Assessment of cost-effectiveness, e.g., in quality-adjusted life years is always a future undertaking and not directly available [15]. However, as the cost-effectiveness must remain open, it is at least clear that additional technological use primarily increases cost and may or may not be "redeemed" in the future.

Shortcomings of this review are the limited number of available comparative studies with an extreme paucity of RCTs, and the lack of comparable financial analyses for the reported open and minimally invasive procedures. Altogether, the role of so-called minimally invasive spinal surgery is far from being defined and Sibylline oracles such as "With education, training, and further research, more of our traditional open surgical management will be augmented or replaced by these technologies and approaches in the future" [10] may possibly turn into a disillusion.

\section{Conclusions}

This review, based solely on the very limited number of available comparative studies, shows no relevant benefit from minimally invasive techniques, and a tendency for more safety in open procedures in lumbar disc herniation, TLIF, and PLIF.

Conflicts of interest None.

\section{References}

1. Arts MP, Brand R, van den Akker ME, Koes BW, Bartels RH, Peul WC, Leiden-The Hague Spine Intervention Prognostic Study Group (SIPS) (2009) Tubular diskectomy vs. conventional microdiskectomy for sciatica: a randomized controlled trial. JAMA 302 (2):149-158

2. Chung RS, Ahmed N (2010) The impact of minimally invasive surgery on residents' open operative experience: analysis of two decades of national data. Ann Surg 251(2):205-212

3. Dhall SS, Wang MY, Mummaneni PV (2008) Clinical and radiographic comparison of mini-open transforaminal lumbar interbody fusion with open transforaminal lumbar interbody fusion in 42 patients with long-term follow-up. J Neurosurg Spine 9(6):560-565

4. Epstein NE (2009) Minimally invasive/endoscopic vs. "open" posterior cervical laminoforaminotomy: do the risks outweigh the benefits? Surg Neurol 71(3):330-331

5. Gepstein R, Shabat S, Reichel M, Pikarsky I, Folman Y (2008) Treatment of postdiscectomy low back pain by percutaneous 
posterior lumbar interbody fusion versus open posterior lumbar fusion with pedicle screws. Spine J 8(5):741-746

6. Harrington JF, French P (2008) Open versus minimally invasive lumbar microdiscectomy: comparison of operative times, length of hospital stay, narcotic use and complications. Minim Invasive Neurosurg 51(1):30-35

7. Kim CW, Lee YP, Taylor W, Oygar A, Kim WK (2007) Use of navigation-assisted fluoroscopy to decrease radiation exposure during minimally invasive spine surgery. Spine J 8(4):584-590

8. Mariscalco MW, Yamashita T, Steinmetz MP, Krishnaney AA, Lieberman IH, Mroz TE (2010) Radiation exposure to the surgeon during open lumbar microdiscectomy and minimally invasive microdiscectomy: a prospective, controlled trial. Spine 36(3):255-260

9. Ntoukas V, Müller A (2010) Minimally invasive approach versus traditional open approach for one level posterior lumbar interbody fusion. Minim Invasive Neurosurg 53(1):21-24

10. Oppenheimer JH, DeCastro I, McDonnell DE (2009) Minimally invasive spine technology and minimally invasive spine surgery: a historical review. Neurosurg Focus 27(3):E9
11. Park Y, Ha JW (2007) Comparison of one-level posterior lumbar interbody fusion performed with a minimally invasive approach or a traditional open approach. Spine 32(5):537-543

12. Peng CW, Yue WM, Poh SY, Yeo W, Tan SB (2009) Clinical and radiological outcomes of minimally invasive versus open transforaminal lumbar interbody fusion. Spine 34(13):1385-1389

13. Perez-Cruet MJ, Fessler RG, Perin NI (2002) Review: complications of minimally invasive spinal surgery. Neurosurgery 51(5 Suppl):S26-S36

14. Schizas C, Tzinieris N, Tsiridis E, Kosmopoulos V (2009) Minimally invasive versus open transforaminal lumbar interbody fusion: evaluating initial experience. Int Orthop 33(6):1683-1688

15. Smith HE, Rihn JA, Brodke DS, Guyer R, Coric D, Lonner B, Shelokov AP, Currier BL, Riley L, Phillips FM, Albert TJ (2009) Spine care: evaluation of the efficacy and cost of emerging technology. Am J Med Qual 24(6 Suppl):25S-31S

16. Villavicencio AT, Burneikiene S, Roeca CM, Nelson EL, Mason A (2010) Minimally invasive versus open transforaminal lumbar interbody fusion. Surg Neurol Int 1:12 